\title{
Predicting Intelligibility: An Investigation of Speech Sound Accuracy in Childhood Apraxia of Speech
}

\author{
KYRA SKOOG, BA ${ }^{1}$; EDWIN MAAS, PHD ${ }^{1}$ \\ ${ }^{1}$ Department of Communication Sciences and Disorders, College of Public Health, Temple University \\ Correspondence: kjskoog@wisc.edu (Kyra Skoog)
}

\begin{abstract}
Background: Childhood apraxia of speech (CAS) is a pediatric speech disorder that significantly affects communication and life participation. Most CAS treatment research uses speech accuracy as primary outcome measure, on the assumption that accuracy predicts communicative success. However, this relationship bas not yet been examined in this population, limiting our understanding of the impact of available treatments. Purpose: The purpose of this study is to explore the relationship between speech accuracy and intelligibility in children with CAS. Intelligibility is defined here as the proportion of words correctly understood by an unfamiliar listener. Methods: Adult listeners, who were unfamiliar with children with $C A S$, listened to recordings of children with $C A S$ producing single words, and typed what they heard the child say. Separately, and prior to the listening experiment, the children's words were scored for accuracy using various measures, including the percent phonemes (sounds) correct (PPC), percent consonants correct (PCC), and percent vowels correct (PVC). The relationship between these accuracy measures and intelligibility were examined descriptively. Results: Preliminary findings suggest that there is a positive relationship between intelligibility and PPC and PCC in children with CAS. Conclusions: Implications of these findings for clinical practice as well as future treatment research are discussed.
\end{abstract}

\section{Introduction}

\section{Childhood Apraxia of Speech (CAS)}

Childhood apraxia of speech (CAS) is a pediatric speech disorder that can significantly hinder communication as well as literacy development and academic success. ${ }^{1-2}$ It is estimated to occur in between 1 to 10 per 1,000 children, ${ }^{3}$ meaning that there are between 40,000 and 400,000 children with CAS in the United States. ${ }^{3-4}$ CAS is thought to reflect a disruption of the complex processes required for planning speech movements, rather than neuromuscular problems such as paralysis of oral articulators (e.g., tongue, lips) or lack of knowledge of the sounds that make up words. ${ }^{5}$ CAS is characterized by inconsistent errors on vowels and consonants, difficulties with coarticulation, and prosodic abnormalities. ${ }^{2,5-7}$ Other traits that may be affected in children with CAS involve cognitive functions such as forming, storing, and representations of auditory/perceptual information. ${ }^{1-2,8-9}$ 


\section{Treatment for Childhood Apraxia of Speech}

Current treatments for CAS with the strongest evidence base are behavioral (speech therapy) and utilize motor-based treatment approaches that target the accuracy of speech productions. These treatments follow principles of motor learning ${ }^{10}$ and rely on frequent, intensive, and individualized treatment sessions that maximize the amount of practice attempts. ${ }^{1-2,8}$ Treatment approaches with controlled empirical evidence include integral stimulation treatment, ${ }^{6-7,}$,11-13 Rapid Syllable Transition (ReST) treatment, ${ }^{14-15}$ ultrasound feedback treatment, ${ }^{17}$ and Prompts for Restructuring Oral Muscular Phonetic Treatment (PROMPT). ${ }^{8,17}$ See Murray et al. ${ }^{18}$ and Maas et al. ${ }^{2}$ for reviews.

However, it is important to note that in virtually all studies that have examined the efficacy of these treatment approaches, the primary outcome measures have focused exclusively on accuracy of speech, typically judged by trained research personnel. With very few exceptions, ${ }^{8}$ studies have not included measures of communicative ability or life participation, which may be considered more functional in nature. As a result, the impact of the various treatments for CAS remains poorly understood, limited by relatively narrow outcome measures.

\section{Functional Outcome Measures}

Intelligible speech is a critical element of successful communication and can be defined as the degree to which a listener can identify words from a spoken signal. ${ }^{19-21}$ One factor that is likely to have an effect on intelligibility is the accuracy of speech productions..$^{22}$ As mentioned above, the evidence base regarding treatment for CAS typically focuses on the impairment level as defined by the World Health Organization's International Classification of Function, Disability, and Health (WHO ICF), ${ }^{23}$ with typical outcome measures based on speech accuracy (e.g., whether a word contains incorrectly produced sounds) rather than functional elements of communication. ${ }^{1,8,24}$

The WHO developed the ICF to act as a framework for describing information on functioning and disability. ${ }^{23}$ The ICF model uses a multi-dimensional approach that defines function on three levels: body functions and structure, activities, and participation ${ }^{23}$ (see Figure 1 for illustration of application of the ICF to CAS). Function at these levels is further influenced by environmental and personal factors. The body structures and functions level refers to the physical, physiological, and cognitive processes that underlie a function, and challenges at this level are referred to as impairments. For instance, a leg fracture represents an impairment of a body structure, and a speech motor planning deficit represents an impairment of a specific cognitive process that disrupts movement of articulators. The activity level refers to the integrated functional activity for which the body structures and functions are used, such as walking or producing speech. Challenges at this level are referred to as limitations; a broken leg may limit the ability to walk, and a speech motor planning impairment may limit the ability to communicate via spoken acoustic signals. The participation level refers to the use of the integrated functional activity to accomplish tasks in daily life and participate in society. Challenges at this level are referred to as restrictions; a limitation in walking may restrict the ability to go grocery shopping, work, or attend social events, and a limitation of speaking ability may restrict the ability to hold important phone conversations, conduct a job interview, or share personal experiences. These levels interact with one another, and with personal and environmental factors, to determine the functional capacities and limitations. Personal factors refer to attitudes and beliefs of the individual, and these may influence the impact of an impairment. For example, a child with a severe impairment and a high level of confidence or strong coping skills may be less restricted in participation than a child with a mild impairment and a low level of confidence or poor coping skills. Environmental factors refer to attitudes and beliefs and settings outside the individual that may affect the restrictions and well-being of the individual, as well as systemic factors such as availability 
of health care and insurance. A supportive environment (e.g., available and accessible health care, positive social network of accepting peers) may mitigate the impact of an impairment, whereas unsupportive environments may exacerbate the impact (e.g., no access to health care, bullying by peers for being different).

Although the ICF model states the importance of weighing each dimension equally, current CAS treatment research favors body functions and structures over the other factors in terms of outcome measures, emphasizing the impairment of an individual.6, 24 However, for children and their families, the ultimate goal is often to be able to communicate successfully ${ }^{1,19-20,25}$ - i.e. family members are most often concerned with the activity and participation levels.

The majority of speech-oriented treatments for CAS make the (usually implicit) assumption that if overall accuracy and consistency improve, then the improvements will carry over to intelligibility. $22,26-27$ This relationship between speech accuracy and intelligibility has been studied in other speech disorders. ${ }^{26,28}$ For example, for children with dysarthria secondary to cerebral palsy, none of the measures of accuracy (e.g., percent consonants correct [PCC], percent vowels correct [PVC], percent whole-word accuracy [PWWA]) correlated significantly with word intelligibility. ${ }^{28}$ However, in this same study, PCC and percent phonemes correct (PPC) did correlate with sentence intelligibility, whereas PVC did not. Additionally, irregularity in productions (e.g., of articulatory errors, speaking rate, loudness variation) has been shown to affect intelligibility in dysarthric speakers, ${ }^{29}$ and vowel acoustics were shown to account for about $45 \%$ of the variation in intelligibility for adults with dysarthria secondary to amyotrophic lateral sclerosis. ${ }^{30}$ Such findings suggest that regularity and vowel accuracy may be important factors in determining the relationship between accuracy and intelligibility. Although the relationship between accuracy and intelligibility has begun to be examined in other speech disorders, this relationship has not been studied in children with CAS. Given the different specific aspects of speech in CAS, including inconsistent distortions of both vowels and consonants and abnormal prosody, it is possible that the relationship between accuracy and intelligibility is different from that observed in other speech disorders.

Figure 1. The World Health Organization International Classification of Function, Disability, and Health (WHO ICF), ${ }^{23}$ as applied to CAS.

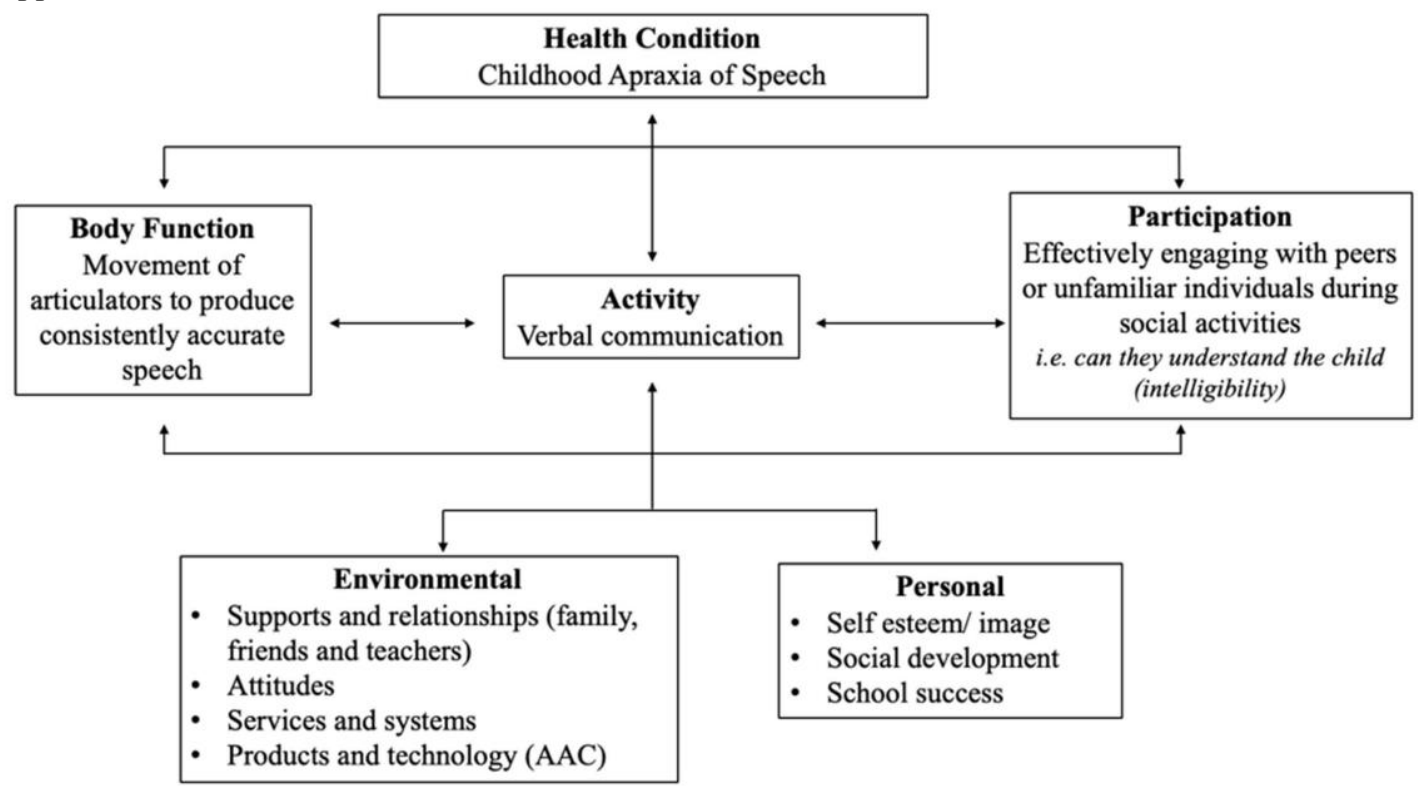




\section{The Present Study}

The primary purpose of the present study was to examine the relationship between intelligibility and various measures of speech accuracy, in order to evaluate the implicit assumption that accuracy measures can be used as proxies for functional measures in CAS. This is important because, if treating at the impairment level does not produce improvements at the activity/participation level, existing treatments may need to be modified, or new treatments must be developed to induce functional gains. A secondary purpose is to examine the relationship between certain speech sounds (consonants and vowels) and intelligibility, in order to better understand the relative contributions of consonants and vowel accuracy to intelligibility in children with CAS. This information may be important for speech-language pathologists in terms of the focus of their treatment, specifically with respect to target selection and focus of feedback and cues. In sum, the study addresses the following two research questions:

1. Is there a relationship between accuracy and intelligibility in children with CAS?

2. What sounds (consonants or vowels), if any, have a larger impact on intelligibility?

To address these questions, we obtained measures of consonant accuracy, vowel accuracy, and phoneme accuracy, from children with CAS. We then obtained intelligibility measures from adult listeners, who were unfamiliar with CAS, based on these speech samples and examined the relationship between the measures. This study is based on speech recordings made in the context of a treatment study, the primary data of which are published elsewhere. ${ }^{6}$ Here we used baseline recordings from three of those children.

\section{Participants}

\section{Speakers}

Three children, all male, aged 5;11 (years; months), 5;11, and 7;11 from a treatment study published elsewhere ${ }^{6}$ participated in this study (see Table 1 for information). Children with CAS were recruited through postings to the Apraxia Kids organization website and at local outreach events. Diagnoses of CAS were made based on independent judgments from four speech-language pathologists (SLPs) with extensive experience in CAS. Each SLP reviewed the evaluation (live or from video) and assigned a score of 0 (no CAS), 1 (possible CAS), or 2 (CAS). A diagnosis of CAS required a mean $>1$ across SLPs. Evaluation included a case history as well as formal and informal measures to evaluate speech, language, and cognitive skills. All testing and treatment were administered by an experienced SLP and took place in a quiet, child-friendly room in the Speech, Language, and Brain Lab at Temple University. The number of sessions required to determine eligibility varied depending on how many sessions were needed to complete the tasks used to diagnose CAS; sessions typically lasted 60 minutes or less. All sessions were audio and video recorded for later analysis. Parent permission was obtained for all children, and all children provided assent. All study procedures were approved by the Temple University Institutional Review Board.

To be included in the study, children had to meet the following criteria: (1) be between the ages of 4 and 12 years, (2) use English as the primary language at home and in school, (3) have communicative intent and output of over 50 words as determined by an SLP and parent report, (4) presence of a speech sound disorder determined by having a score below the 10th percentile on the Goldman-Fristoe Test of Articulation- Second Edition ${ }^{31}$; (5) have CAS as the primary speech diagnosis as determined by SLP rating and an Apraxia Score of 1 or 2 on the Maximum Performance Task protocol, the only prospectively validated diagnostic procedure for CAS with sensitivity and specificity $>90 \% ; 32-34$ (6) have normal hearing based on parent report; and (7) have age-appropriate nonverbal cognitive function, as determined by a 
T-score within 1.5 SDs of the mean on nonverbal subtests of the Reynolds Intellectual Assessment Scales. ${ }^{35}$ The following criteria would result in exclusion form the study: (1) diagnosis of social/pragmatic disorders (e.g., autism), as per referral diagnosis; (2) uncorrected vision impairments (per parent report) that would likely interfere with the ability to take advantage of visual cues; (3) significant impairments of oral structure (e.g., cleft palate) as judged by the SLP based on an oral motor examination; ${ }^{36}$ and (4) a primary diagnosis of dysarthria (a speech disorder due to neuromuscular impairments in strength, range of motion, or speed of movement), as judged by the SLP and a Dysarthria Score of 2 on the Maximum Performance Task protocol. ${ }^{33-34}$

Descriptive measures (not used to determine inclusion/exclusion) were also obtained. These measures included receptive vocabulary (Peabody Picture Vocabulary Test, 4th edition) ${ }^{37}$, expressive vocabulary (Expressive Vocabulary Test, 2nd edition), ${ }^{38}$ speech error inconsistency (Diagnostic Evaluation of Articulation and Phonology). ${ }^{39}$

Table 1. Participant information.

\begin{tabular}{lcccccccccc}
\hline ID & Sex & Age & GFTA $^{a}$ & DEAP $^{b}$ & EVT $^{c}$ & PPVT $^{d}$ & RIAS $^{e}$ & MaxPT Dys $^{f}$ & MaxPT CASs $^{\prime}$ & CAS Dx $^{\mathrm{h}}$ \\
\hline $\mathbf{3 2 1}$ & M & $5 ; 11$ & 41 & $72 \%$ & 84 & 82 & 48 & 0 & 2 & $1.50(0.58)$ \\
$\mathbf{3 2 2}$ & M & $5 ; 11$ & 47 & $52 \%$ & 88 & 92 & 43 & 0 & 2 & $1.75(0.50)$ \\
$\mathbf{3 2 3}$ & M & $7 ; 11$ & 55 & $80 \%$ & 88 & 89 & 42 & 0 & 2 & $1.75(0.50)$
\end{tabular}

${ }^{a}$ Goldman-Fristoe Test of Articulation, $2^{\text {nd }}$ ed.$^{31}$ standard score (normative mean $=100, \mathrm{SD}=15$ ); ${ }^{\mathrm{b}}$ Diagnostic Evaluation of Articulation and Phonology ${ }^{39}$ Word Inconsistency Subtest percent inconsistent (typically developing children score $<10 \%)^{40}$; ${ }^{c}$ Expressive Vocabulary Test, $2^{\text {nd }}$ ed. ${ }^{38}$ standard score (normative mean $=$ $100, \mathrm{SD}=15) ;{ }^{\mathrm{d}}$ Peabody Picture Vocabulary Test, $4^{\text {th }}$ ed. ${ }^{37}$ standard score (normative mean $\left.=100, \mathrm{SD}=15\right) ;{ }^{\mathrm{e}}$ Reynolds Intellectual Assessment Scales ${ }^{35}$ nonverbal cognition composite T-score (normative mean $=50, \mathrm{SD}=$ 10); ${ }^{\mathrm{f}}$ Maximum Performance Test Protocol ${ }^{32-33}$ dysarthria score; $\mathrm{g}$ Maximum Performance Test Protocol ${ }^{32-33}$ apraxia of speech score; ${ }^{\mathrm{h}}$ Mean (standard deviation) of four expert SLP ratings $(0=$ no CAS, $1=$ possible CAS, 2 $=\mathrm{CAS})$.

\section{Listeners}

In addition to the child participants, nine adult participants ( 8 women, 1 man) were recruited through a summer undergraduate research program at Temple University to act as unfamiliar listeners. Inclusion criteria for adult listeners included (1) no known hearing disorder, as determined by a hearing screening and self-report, (2) fluent English speaker, per self-report, and (3) no prior knowledge of the GFTA or children with CAS, per self-report. The mean age was 21.4 years $(S D=0.9$; range $=20-23)$. Adult participants provided consent and were given monetary compensation for their participation. All study procedures were approved by the Temple University Institutional Review Board.

\section{Design}

The intelligibility experiment included speech samples from the three children with CAS. Each child's speech samples were transcribed by three independent listeners, resulting in nine listeners. Each listener only heard a speech sample from one child. Assignment of listeners to speech samples was randomized. Words were presented in a different random order for each listener. 


\section{Tasks and Procedures}

Speech Sample Collection

The speech samples used in this study were obtained from the GFTA-2. ${ }^{31}$ The GFTA-2 is a standardized picture-naming test consisting of 53 common words familiar to most children (e.g., house, duck, yellow) that assesses an individual's articulation of consonants and consonant clusters of Standard American English in all allowable word positions (e.g., /k/ occurred in initial, medial, and final position in the words 'cup', 'monkey', and 'duck'). The pictures included in the assessment are designed to be common words and objects that are age appropriate and not specific to one culture. The clinician is allowed to provide prescribed cues as needed (e.g., What is this part of the house? 'window'; Do you know the long word for it? 'airplane'). The test is designed for individuals ages 2 to 21 and typically takes between 5 and 15 minutes to administer (depending on age and ability). The child's responses are recorded on a paper scoresheet that documents any errors the child may have made. For this study, children's responses were also audio recorded using a high-quality vocal condenser microphone at $44.1 \mathrm{kHz}$. The GFTA-2 renders a raw score and a standard score based on the child's accuracy of a subset of consonants in the words. ${ }^{31}$ However, for present purposes, we examined accuracy of all consonants and all vowels in the 53 words to provide a more complete measure of accuracy. Specific measures are described below (see Analysis).

Speech Sample Preparation for Listeners

Words from the GFTA were edited from session recordings into individual sound files by a trained research assistant using Adobe Audition 2020 on a Dell desktop computer. Each individual word was isolated to not include the clinician cue or feedback from the clinician, or extraneous sounds (e.g., coughs, yawns, background noise). Some words were excluded from the study due to recording problems such as background noise, clinician speaking over production, noise made by toys or silly voices that would hinder accuracy, stress, or prosody of responses. If another attempt of the word was available with acceptable recording quality, this other attempt was used instead. Words that were excluded from the listening experiment for these reasons were also excluded from the accuracy calculations (described below). Individual sound files were amplitude-normalized to ensure consistent volume throughout the experiment.

\section{Listening Experiment}

Individuals completed the intelligibility testing in a quiet room in the Speech, Language and the Brain Lab at Temple University. Prior to testing, the adult participants were given consent forms to explain the project and a hearing screening to confirm that they met inclusion criteria. Once the hearing screening concluded, the experiment started. The study was controlled by the E-Prime software (v2.0: Psychology Software Tools Inc.) running on a Dell desktop computer. Each listener was presented with audio clips of words produced by one of the children with CAS through a set of external Logitech speakers. Prior to hearing child utterances, participants completed a practice trial in which they heard three different words said by adults with 100\% intelligibility and accuracy to help explain the format of the study as well as adjust the sound level to a comfortable volume. The order of words was randomized for each listener to avoid any systematic effects of fatigue, boredom, or increasing familiarity with the child's speech within a session. Listeners were instructed to type in what they heard the child say even if they perceived it to be a non-word. Listeners were permitted to hear each word twice and were prompted to type a response after each time the word was played. The study was completed in a single block consisting of approximately 43 words (see Table 2) and concluded when the listener had heard all words produced by the child. Sessions lasted an average of 45 minutes and were never longer than 55 minutes. 


\section{Analysis}

\section{Accuracy Scoring}

Accuracy was scored prior to the listening experiment and was based on the child's first response when possible. The reason to use the first response is that the child's first response best reflects the child's ability to independently construct the movement plan for the utterance, without opportunity to revise. In some cases, children said the target word multiple times. In these situations, the first attempt was used unless the first response was not usable, for example due to background noise, simultaneous speech from the clinician, or poor recording quality. In these cases, the next attempt was included if available and usable. Accuracy measures were scored by trained research assistants who were unfamiliar with the children and were not involved in the treatment study.

Four measures of accuracy were calculated: (1) percent consonants correct (PCC), (2) percent vowels correct (PVC), (3) percent phonemes (speech sounds) correct (PPC), and (4) percent whole word accuracy (PWWA). PCC was calculated by taking the number of consonants correctly produced divided by the total number of consonants in the target words. ${ }^{41}$ PVC followed the same calculation as PCC but using the number of vowels instead of consonants. PPC was calculated by dividing the number of correctly produced sounds (consonants and vowels) by the total number of sounds in the target words. For PCC, PVC, and PPC, sounds were considered incorrect if the child substituted a different sound for the target, deleted the target sound, or distorted it. For PWWA, ${ }^{28}$ words were scored as 0 (error in word production) or 1 (no error in word), and the number of words without errors was divided by the total number of words.

Intelligibility

To determine intelligibility, listener responses were first scored on a binary scale $(0=$ does not match target; 1 = matches target). If the listener gave more than one response to a recording, the second response was used to calculate intelligibility, regardless of match (e.g., target 'drum', trial 1: drum, trial 2: gum; response would be scored 0 ). The reason to use the second response is that it represents the listener's final interpretation of the child's message, and thus more closely resembles intelligibility in a natural environment. Trained lab assistants judged whether the orthographic responses matched the target word. Care was taken to ensure that spelling errors did not affect measured intelligibility. Words were considered incorrect if misspelled, except when it was clearly a typing error (e.g., switching the order of letters such as 'huose' for the target 'house') or the misspelled word was homophonous with the target (e.g., typing 'waggon' for the target 'wagon'). In most cases, listener responses were considered incorrect if they contained multiple words. However, some child speakers inserted "a" before noun targets that could not be edited out (e.g., 'a house' for 'house'). When this occurred, a note was made prior to testing so that they could be accounted for; if a listener typed 'a house' this was considered a match (score of 1). For each child, mean intelligibility was then computed by dividing the number of matching responses by all target words. For each child, the intelligibility scores were averaged across the three listeners. These average intelligibility scores were used for analysis.

\section{Statistical Analysis}

The average intelligibility scores were plotted against the PPC, PVC, PCC, and PWWA scores. Given the small sample size in this preliminary study, no formal statistical analysis (e.g., correlation) was undertaken; instead, data were examined visually. We predicted that there would be a positive relationship between accuracy and intelligibility, regardless of the accuracy rating method. 


\section{Results}

Data for all children is provided in Table 2. Scatterplots for each error measure against intelligibility is provided in Figure 2.

Table 2. Child data for the accuracy measures and intelligibility. PCC = Percent Consonants Correct; PVC $=$

Percent Vowels Correct; PPC $=$ Percent Phonemes Correct; PWWA $=$ Percent Whole-Word Accuracy.

\begin{tabular}{ccccccc}
\hline Child ID & PCC & PVC & PPC & PWWA & Number of words included & Mean \% intelligibility \\
\hline 321 & 36 & 76 & 50 & 0 & 42 & 15.1 \\
322 & 49 & 81 & 60 & 4 & 42 & 29.3 \\
323 & 75 & 71 & 74 & 26 & 44 & 52.0 \\
\hline
\end{tabular}

Figure 2. Scatterplots of intelligibility against Percent Consonants Correct (PCC; top left), Percent Vowels Correct (PVC; top right), Percent Phonemes Correct (PPC; bottom left), and Percent Whole Word Accuracy (PWWA; bottom right).
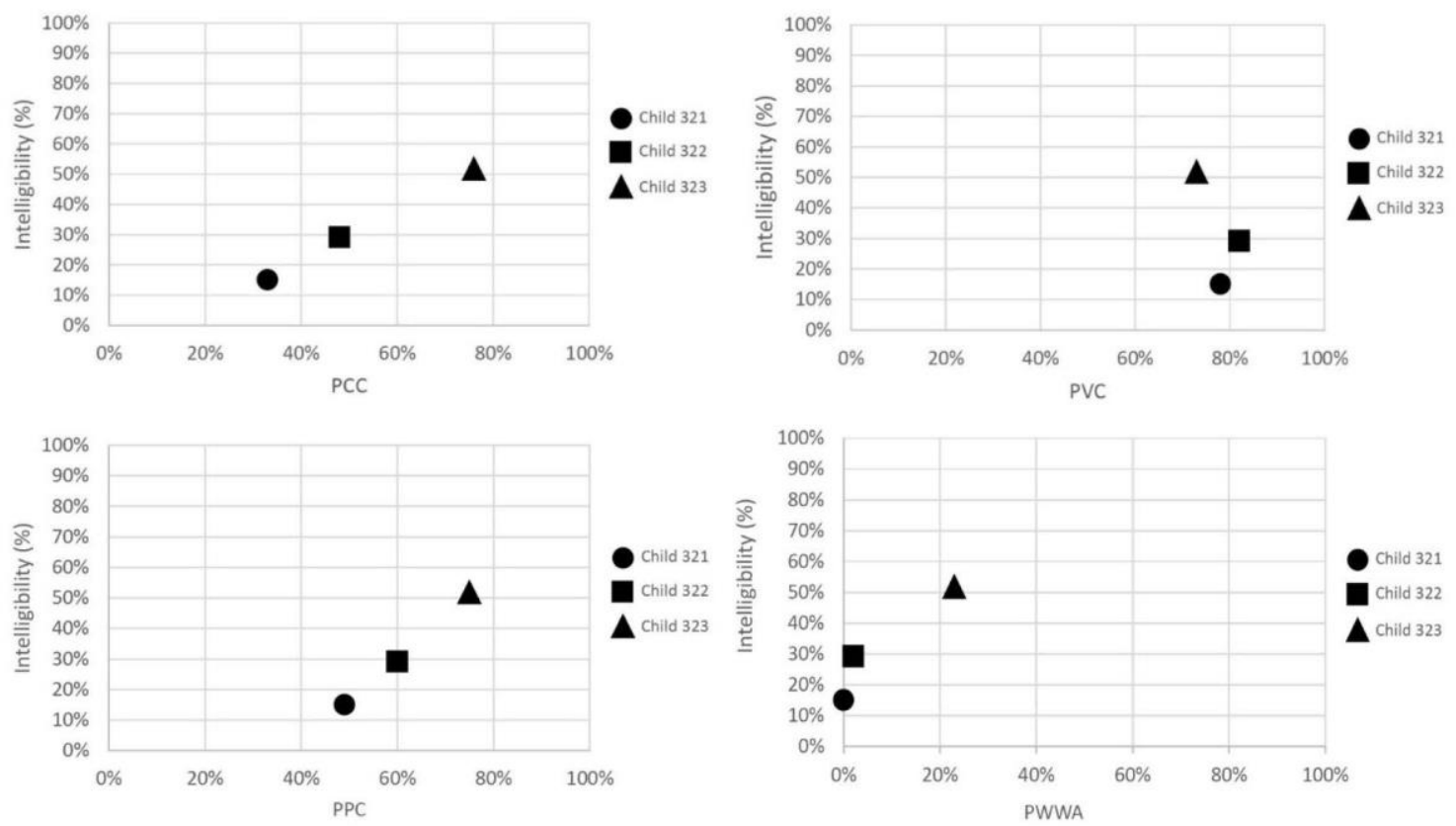

\section{Discussion}

The goal of this study was to investigate the relationship between accuracy and intelligibility in children with CAS, and to determine which sounds contribute most to intelligibility. These preliminary data show a positive relationship between some measures of accuracy and intelligibility. In particular, measures that include consonant accuracy (PCC and PPC) and whole-word accuracy appear to be predictive of intelligibility. By contrast, there was no clear relationship between PVC and intelligibility among these three children with CAS. Below we discuss the findings and their implications. 


\section{Speech Sound Accuracy and Intelligibility}

PPC, PCC, and PWWA all showed a positive association with intelligibility, indicating that as accuracy increases, so does intelligibility. This is congruent with the hypothesis and previous studies that examined this relationship with other motor speech disorders. ${ }^{28}$ This finding is important because it suggests that the impairment-level primary outcome measures of accuracy typically used in clinical trials for CAS do capture at least some relevant aspects of the more functional activity level of function in children with CAS. This supports the relevance of the current evidence base regarding impact of treatment, and is encouraging for critical stakeholders, notably children and their families as well as the SLPs who work with them. These findings support the continued use of accuracy measures in clinical trials for CAS. However, it is also important to note that the relationship between accuracy and intelligibility was not perfect, indicating that other factors are at play in intelligibility that are not captured by accuracy measures. As such, further research is needed to better understand the relationship between impairment-level measures and activity- and participation-level measures in children with CAS. For this reason, future clinical trials of CAS should also incorporate measures of intelligibility and communicative participation to fully understand and establish the functional impact of treatment.

Interestingly and importantly, the present data suggest that vowel accuracy does not contribute to intelligibility of speech. This finding is consistent with findings of Hodge et al. ${ }^{28}$ with children with dysarthria, but was somewhat surprising given that children with CAS often produce vowel errors (as did the three children here) and that research with other populations has suggested that vowels contribute substantially to intelligibility. ${ }^{30}$ One possible reason is that vowel accuracy was sufficiently high in these children, and that intelligibility is only impacted when vowel accuracy drops below a certain level (e.g., PVC $<50 \%$ ). The three children here all had PVCs greater than 70\%. Another potential reason for the lack of relationship between PVC and intelligibility is the limited variability in vowel accuracy (range of only 10\% across children), which makes it difficult to detect relationships. Future research with a larger sample of children and greater range of PVC scores is needed to determine whether the lack of a relationship between PVC and intelligibility is due to this lack of variability or whether vowel accuracy does not play a significant role in intelligibility in CAS.

\section{Clinical Implications}

As previously stated, the data presented here are preliminary and must be considered with caution given the small sample size. However, if these results are confirmed in studies with larger sample sizes, these results can inform treatment decisions and potentially improve the treatment of CAS. In particular, if this pattern is replicated, then the findings may indicate that while vowels are important for diagnostics, consonants may be more important to target in treatment given their greater impact on intelligibility. This may guide target selection as well as the focus of cues and feedback on consonants rather than vowels during speech therapy. More generally, these findings do suggest that at least certain measures of accuracy may predict intelligibility to some degree, providing some tentative support for the implicit assumption in treatment studies that improved accuracy is likely to be associated with improved intelligibility. This means that practicing SLPs can have some degree of confidence that the currently available evidence base is relevant with respect to the goal of making a meaningful and functional impact on the lives of children with CAS and their families.

\section{Future Directions}

To the best of our knowledge this study represents the first published investigation of the relationship between accuracy and intelligibility in CAS. Although the findings are interesting and promising, they are 
preliminary and based on a small sample size of children ranging from 5;11 to 7;11, and with no normal statistical analysis. Future studies are needed to replicate and expand this work and investigate other aspects of accuracy and intelligibility in children with CAS. In addition to a larger sample size of children with a greater range of PVC, future research should be extended to examine the relationship between accuracy and intelligibility of multiword sequences (e.g., sentences), the relationship between different classes of consonants and intelligibility, or intelligibility of words while imbedded in a sentence. Furthermore, inclusion of both accuracy and intelligibility measures in treatment studies for CAS would enable a more powerful examination of this relationship, by examining the correlation between change in accuracy and change in intelligibility. Finally, future research should examine this relationship more systematically across a wider age range.

\section{Conclusion}

In conclusion, this study shows that there is a relationship between speech accuracy and intelligibility in children with CAS. Of the speech sounds assessed, consonants appeared to have the greatest impact on intelligibility, whereas there was no clear relationship between intelligibility and vowel accuracy. Future studies are needed to replicate and extend these findings, but the present preliminary data suggest that the use of impairment-level outcome measures in clinical trials for CAS is justified in that they predict more functional measures, although incorporation of functional measures in treatment studies is highly recommended to fully understand the relationship between accuracy and intelligibility and the functional impact of treatment. Finally, if these findings are replicated, they also hold important implications for clinical practice, in that SLPs may expect the greatest functional gains by targeting consonant accuracy rather than vowel accuracy in treatment.

\section{Disclosures and Conflicts of Interest}

EM receives salary from Temple University as a faculty member. He also serves as Editorial Board member of CommonHealth. The findings of this study were presented at the Temple University College of Public Health Research and Evidence-Based Practice Day (April 2020, Philadelphia, PA).

\section{Funding Sources}

This research was supported by a Diamond Research Scholarship awarded to KS, a Students Preparing for Academic Research Careers (SPARC) award to KS from the American Speech-Language-Hearing Association, and a research grant from Apraxia Kids to EM. The findings and views expressed here do not necessarily represent the views of these organizations.

\section{Acknowledgments}

We thank the children and their families, as well as the adults who participated for their time and effort. We also thank Hannah Braid, Michelle Ferguson, and Kelly Sauer for their assistance with preparing the speech error data.

\section{References}

1. Murray E, Iuzzini-Seigel J. Efficacious treatment of children with childhood apraxia of speech according to the international classification of functioning, disability and health. Perspect ASHA Spec Interest Groups, 2017;2(2): 61-76. 
2. Maas E, Gildersleeve-Neumann CE, Jakielski KJ, Stoeckel R. Motor-based intervention protocols in treatment of childhood apraxia of speech (CAS). Curr Dev Dis Rep, 2014; 1(3): 197206.

3. Shriberg LD, Aram DM, Kwiatkowski J. Developmental apraxia of speech: I. Descriptive and theoretical perspectives. J Speech Lang Hear Res, 1997; 40(2): 273-285.

4. United States Census Bureau. Children Characteristics: total number of children under 18 years of age. 2018. Retrieved from https://data.census.gov/

5. American Speech-Language-Hearing Association. (2007). Childhood apraxia of speech [Technical Report]. Retrieved from www.asha.org/policy

6. Maas E, Gildersleeve-Neumann C, Jakielski K, Kovacs N, Stoeckel R, Vradelis H, Welsh M. Bang for your buck: A single-case experimental design study of practice amount and distribution in treatment for childhood apraxia of speech. J Speech Lang Hear Res, 2019;62(9): 3160-3182.

7. Strand EA, Stoeckel R, Baas B. Treatment of severe childhood apraxia of speech: A treatment efficacy study. J Med Speech-Lang Pat, 2006;14:297-307.

8. Namasivayam AK, Pukonen M, Goshulak D, Hard J, Rudzicz F, Rietveld T, ... Van Lieshout P. Treatment intensity and childhood apraxia of speech. Int J Lang Commun Dis, 2015; 50(4): 529546.

9. Shriberg LD, Lohmeier HL, Strand EA, Jakielski KJ. Encoding, memory, and transcoding deficits in childhood apraxia of speech. Clin Linguist Phonet, 2012; 26: 445-482

10. Maas E, Robin DA, Austermann Hula SN, Freedman SE., Wulf G, Ballard KJ, Schmidt RA. Principles of motor learning in treatment of motor speech disorders. Am J Speech-Lang Pat, 2008;17(3):277-298.

11. Edeal DM., Gildersleeve-Neumann CE. The importance of production frequency in therapy for childhood apraxia of speech. Am J Speech-Lang Pat, 2011;20: 95-110.

12. Maas E, Butalla CE, Farinella KA. Feedback frequency in treatment for childhood apraxia of speech. Am J Speech-Lang Pat, 2012; 21:239-257

13. Strand EA, Debertine P. The efficacy of integral stimulation intervention with developmental apraxia of speech. J Med Speech-Lang Pat, 2000;8(4): 295-300.

14. Ballard KJ, Robin DA, McCabe P, McDonald J. A treatment for dysprosody in childhood apraxia of speech. J Speech Lang Hear Res, 2010; 53:1227-1245.

15. Murray E, McCabe P, Ballard KJ. A randomized controlled trials for children with childhood apraxia of speech comparing Rapid Syllable Transition treatment and the Nuffield Dyspraxia Programme-Third Edition. J Speech Lang Hear Res, 2015;58: 669-686.

16. Preston JL, Leece MC, McNamara K., Maas E. Variable practice during ultrasound visual feedback treatment for childhood apraxia of speech. Am J Speech-Lang Pat, 2017; 26:840-852.

17. Dale PS, Hayden DA. Treating speech subsystems in childhood apraxia of speech with tactual input: The PROMPT approach. Am J Speech-Lang Pat, 2013; 22: 644-661.

18. Murray E, McCabe P, Ballard KJ. A systematic review of treatment outcomes for children with childhood apraxia of speech. Am J Speech-Lang Pat, 2014;23: 486-504.

19. Allison KM, Hustad KC. Impact of sentence length and phonetic complexity on intelligibility of 5-year-old children with cerebral palsy. Int J Speech-Lang Path, 2014; 16(4): 396-407.

20. Allison, KM, and Hustad, KC. Data-driven classification of dysarthria profiles in children with cerebral palsy. J Speech Lang Hear Res, 2018;61(12): 2837-2853.

21. Kent RD, Weismer G, Kent JF, Rosenbek, JC. Toward phonetic intelligibility testing in dysarthria. J Speech Hear Dis, 1989; 54(4): 482-499.

22. Hustad KC, Oakes A, Allison K.. Variability and diagnostic accuracy of speech intelligibility scores in children. J Speech Lang Hear Res, 2015;58(6): 1695-1707.

23. World Health Organization. ICF-CY: International classification of functioning, disability and health: Children and youth version. Geneva: author; 2007. 
24. Kearney E, Granata F, Yunusova Y, Van Lieshout P, Hayden D, Namasivayam A. (2015). Outcome measures in developmental speech sound disorders with a motor basis. Curr Dev Dis Res, 2015;2(3): 253-272.

25. Rusiewicz HL, Maize K., Ptakowski T. Parental experiences and perceptions related to childhood apraxia of speech: Focus on functional implications. Int J Speech-Lang Path, 2018;20(5): 569580.

26. Hodge MM, \& Gotzke CL. Minimal pair distinctions and intelligibility in preschool children with and without speech sound disorders. Clinical Linguistics \& Phonetics, 2011;25(10), 853-863.

27. Hodge MM, \& Gotzke CL. Criterion-related validity of the Test of Children's Speech sentence intelligibility measure for children with cerebral palsy and dysarthria. Int J Speech-Lang Path, 2014;16(4): 417-426.

28. Hodge M, Brown C, \& Kuzyk T. Predicting intelligibility scores of children with dysarthria and cerebral palsy from phonetic measures of speech accuracy. J Med Speech-Lang Path, 2012;20(4), 41-46.

29. Lansford KL, Borrie SA, Barrett TS. Regularity matters: Unpredictable speech degradation inhibits adaptation to dysarthric speech. J Speech Lang Hearing Res, 2019;62(12):4282-90.

30. Turner GS, Tjaden K., Weismer G. The influence of speaking rate on vowel space and speech intelligibility for individuals with amyotrophic lateral sclerosis. J Speech Lang Hear Res, 1995;38(5):1001-1013

31. Goldman R, Fristoe M. Goldman-Fristoe Test of Articulation-Second Edition (GFTA-2). Austin, TX: Pro-Ed; 2000.

32. Rvachew S, Hodge M, Ohberg A. Obtaining and interpreting maximum performance tasks from children: A tutorial. J Speech Lang Path Audiol, 2005; 29:146-157.

33. Thoonen G, Maassen B, Gabreëls F, Schreuder R. Validity of maximum performance tasks to diagnose motor speech disorders in children. Clin Linguist Phon, 1999;13:1-23.

34. Thoonen, G, Maassen B, Wit J, Gabreëls F, Schreuder R. The integrated use of maximum performance tasks in differential diagnostic evaluations among children with motor speech disorders. Clin Linguist Phon, 1996; 10: 311-336.

35. Reynolds CR., Kamphaus RW. Reynolds Intellectual Assessment Scales (RIAS). Lutz, FL: Psychological Assessment Resources; 2003.

36. Robbins J, Klee T. Clinical assessment of oropharyngeal motor development in young children. J Speech Lang Hear Dis, 1987;52:271-277.

37. Dunn LM, Dunn DM. Peabody Picture Vocabulary Test (4th ed.). AGS/Pearson; 2007.

38. Williams K. Expressive Vocabulary Test (2nd ed.). Circle Pines, MN: American Guidance Services; 2007.

39. Dodd B, Hua Z, Crosbie S, Holm A, Ozanne A. Diagnostic Evaluation of Articulation and Phonology. San Antonio, TX: PsychCorp; 2006.

40. Dodd B. Differential diagnosis of pediatric speech sound disorder. Curr Dev Dis Rep, 2014:1(3), 189-196.

41. Shriberg LD, Austin D, Lewis BA, McSweeny JL, Wilson DL. The percentage of consonants correct (PCC) metric: Extensions and reliability data. J Speech Lang Hear Res. 2017;40(4):708722.

\section{Statement of Contributions}

Kyra Skoog helped conceive the idea for this study, helped design the study, prepared stimuli and programmed the intelligibility experiment, collected the intelligibility data, analyzed the data, and drafted the manuscript. 
Edwin Maas helped conceive the idea for this study and design the study, assisted with collecting the speech samples, assisted with data analysis, and provided input on all aspects of the manuscript.

\section{ORCID IDs}

Edwin Maas: ORCID 0000-0003-4452-2196. 